\title{
Color Glasses during Morning Drive for Commuting-Effects on Autonomic Functions, Alertness, and Nocturnal Sleep
}

\author{
Emi Yuda, Yutaka Yoshida, and Junichiro Hayano
}

\begin{abstract}
Studies of illumination suggest possibility of color lights for adjusting our mind and body to suit desired activities. To examine if similar effects can be obtained by modulating relative contents of light color, biological effects of color-glass wearing during early-morning drive for commuting were studied. Compared with clear glasses, blue glasses increased low frequency $(0.04-0.15 \mathrm{~Hz})$ power and deceleration capacity and decreased respiration frequency during driving, while green, orange, and pink glasses made no significant difference from clear glasses. None of the color glasses showed significant difference in the performance to psychomotor vigilance test performed at the beginning and the end of work at job place or in the autonomic functions during sleep of that night. An increase in the content of blue-wavelength light by blue glasses during morning drive may attenuate sympathetic function, but it has no significant after-effect on daytime behavioral alertness or nighttime autonomic functions.
\end{abstract}

Index Terms-Behavioral alertness, blue light, color glasses, heart rate variability, sleep.

\section{INTRODUCTION}

Light has different biological effects with its color. Light affects many physiological parameters such as melatonin, alertness, body temperature, heart rate, and heart rate variability (HRV) and the sensitivities of these functions to light differ depending on its color [1]-[5]. These suggest possibility that by selecting adequate color for environmental lighting, we may be able to adjust our mind and physical states to suit our desired activities, such as work, study, rest, and sleep. However, it seems difficult to respond finely to each individual's different needs and particularly, it is unrealistic to control light environment of a moving person.

To overcome this problem, the present study examined the usefulness of color glasses to control the color of light that enters the eye. Colored glasses can only reduce the photo-spectral components that exist in the environmental light and cannot add or increase any photo-spectral components. In previous studies, however, we observed that exposure to blue light enhances autonomic and behavioral alertness in healthy subjects and that the effects of blue light are greater than white light containing the same amount of blue wavelength light [6]. This finding suggested that the relative content of blue wavelength light is an important

Manuscript received August 30, 2017; revised November 30, 2017. This work was performed as a collaborative study with Tokai Optical Co., Ltd.

The authors are with the Nagoya City University Graduate School of Medical Sciences, Nagoya 467-8602, Japan (e-mail: emi21@med.nagoya-cu.ac.jp, yyoshida@med.nagoya-cu.ac.jp, hayano@med.nagoya-cu.ac.jp). determinant of the arousal effect of light. Because color glasses can modulate the relative contents of photo-spectral component, our approach seems promising. In this study, we used color glasses during early-morning drive for commuting. This is because morning light is a critical cue to reset our circadian clock to environmental time [7] and because the periods of morning drive seemed the most realistic time to wear color glasses among those in our daily activities.

\section{Methods}

\section{A. Subjects}

Subjects of this study were 12 healthy people (age, $40 \pm 8$ [28]-[52] yr, 6 males and 6 females). Inclusion criterion was healthy people with normal color vision who are commuting by car every day. Subjects were excluded if they were pregnant, taking beta-blocker, or had atrial fibrillation, diabetes mellitus under medication, acute illness requiring hospitalization within past 3 months, or malignant disease. All of them gave written informed consent to participate to this study. This study was performed along with the protocol that has been approved by the Ethics Review Committee of Nagoya City University Graduate School of Medical Sciences and Nagoya City University Hospital (\#60160137).
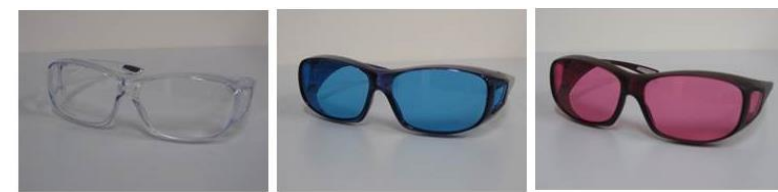

Fig. 1. Clear and color glasses developed for this study (Tokai Optical Co., LTD, Okazaki, Japan).
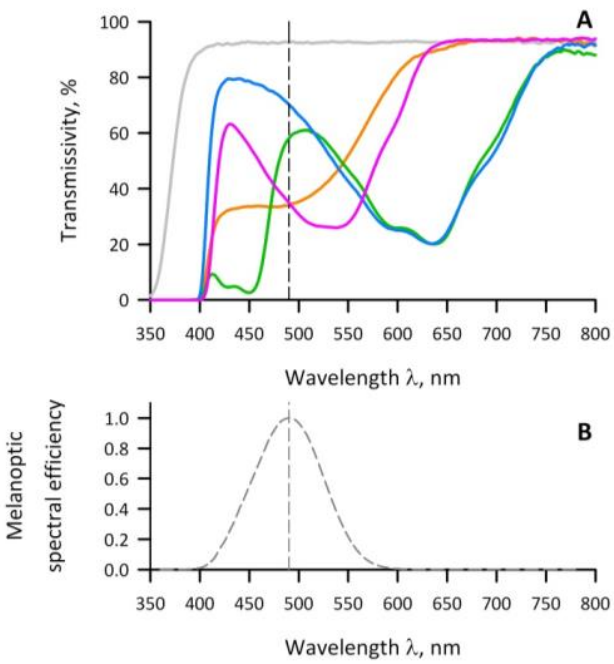

Fig. 2. Transmissivity spectrum of clear and color glasses (A) and spectral efficiency of melanopic component (B). 


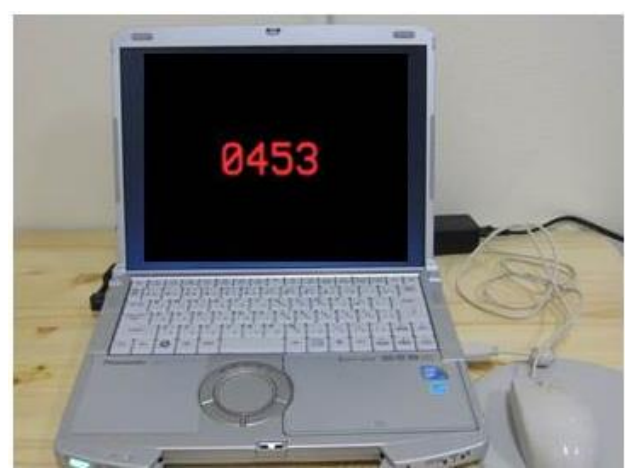

Fig. 3. Psychomotor vigilance test (PVT) with PC-PVT

\section{B. Color Glasses}

For this study, clear glasses and blue, green, orange, and pink color glasses have been developed by Tokai Optical Co., Ltd., Okazaki, Japan for this study (Fig. 1). Fig. 2 shows the transmissivity spectra of the clear and color glasses (provided by the company).

\section{Measurements}

Ambulatory electrocardiogram (ECG) was measured continuously for 24 hours with Holter ECG recorder (Cardy pico 300+, Suzuken Co., Ltd., Nagoya Japan). ECG data were digitized at $125 \mathrm{~Hz}$ and stored in the recorder.

Behavioral alertness was evaluated by psychomotor vigilance test (PVT). Validated software called PC-PVT [8] was used, whose free software was downloaded from http://bhsai.org/downloads/pc-pvt/ and installed in a notebook computer (Let's note CF-S10, Panasonic Co., Osaka, Japan). The anticipation was set at $100 \mathrm{~ms}$, deadline at $65,000 \mathrm{~ms}$, and minor lapse at $500 \mathrm{~ms}$. PVT measures sustained or vigilant attention by recording reaction time (RT) to visual stimuli occurring at random inter-stimulus intervals. The PVT software presented abruptly a time counter on computer display that incremented the number at every $1 \mathrm{~ms}$ (Fig. 3). In this study, the total trial time was set at $300 \mathrm{sec}$, during which 46 to 51 stimuli were presented. According to the earlier studies [9], we measured the number of minor lapse frequency ( $\mathrm{RT} \geq 500 \mathrm{~ms}$ ) with transformation $(\sqrt{x}+\sqrt{x+1})$, fastest and slowest 10th percentile RTs, and difference between fastest and slowest RTs.

\section{Study Protocol}

The scheme of the protocol is presented in Fig. 4. Subjects were instructed to attach ECG electrodes and a recorder on their chest wall in the morning and to continue recording for 24 hours under their daily activities. They were also instructed to wear glasses of a prespecified color during driving for commuting in the morning. When they arrived at their work place in the morning and before they leave for home in the evening, PVT was performed. After coming home, ECG were recorded continuously even during sleep until they got up in the next morning. Then, they detached the ECG electrodes and recorder.

In each subject, measurements with this protocol were repeated for 5 times with differing colors of glasses (clear, blue, green, orange, and pink). The order of colors was counter-balanced among subjects to exclude order effect. Also, in each individual subject, measurements were performed on the same working day of week to minimize the influence of the different work schedules among the days of week. As the results, each subject continued measurement once a week for 5 consecutive weeks.

\begin{tabular}{|c|c|c|c|c|}
\hline Home & $\begin{array}{c}\text { Driving for } \\
\text { attendance }\end{array}$ & Workplace & $\begin{array}{c}\text { Driving for } \\
\text { returning } \\
\text { home }\end{array}$ & Home \\
\hline \multicolumn{1}{|c|}{ Color glasses } & $\begin{array}{l}5 \text { min } \\
\text { PVT }\end{array}$ & $\begin{array}{l}5 \text { min } \\
\text { PVT }\end{array}$ \\
\hline \multicolumn{4}{|c|}{ Holter ECG monitoring } \\
\hline
\end{tabular}

Fig. 4. Schema of study protocol.
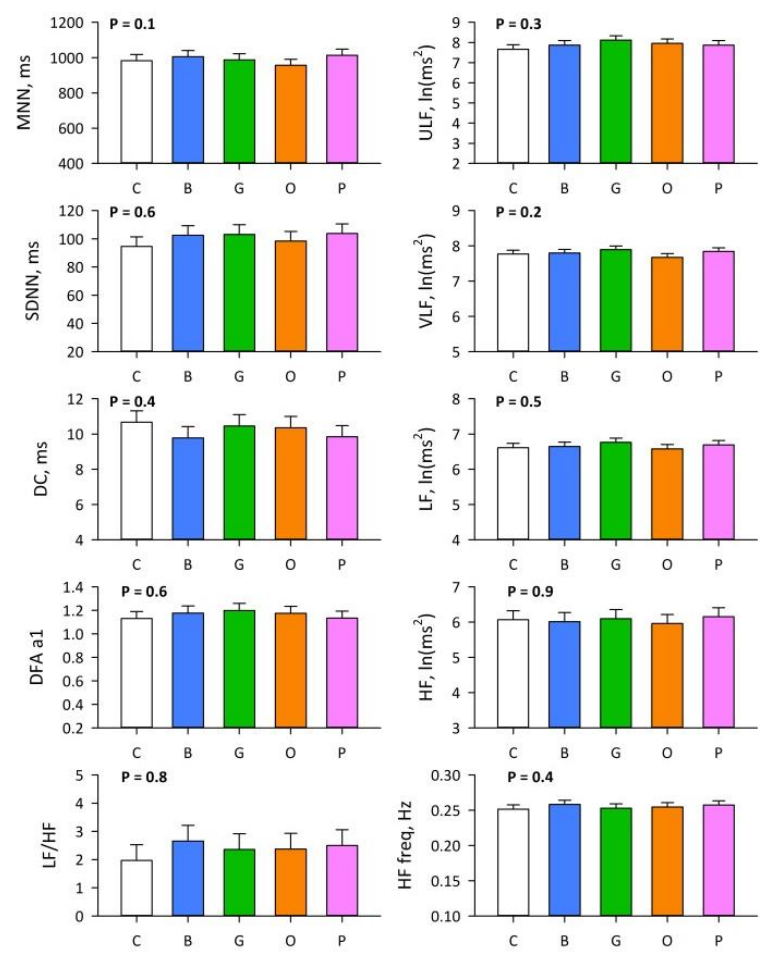

Fig. 5. Effects of color glasses on HRV measures during early morning drive for commuting. P values indicate significance of the effect of glass color.

\section{E. Data Analysis}

Digitized ECG data were analyzed off-line on a personal computer. The temporal positions of all QRS waves were detected with a fast-peak detection algorithm. After all errors in the detection of QRS waves were edited, time series of the $\mathrm{R}-\mathrm{R}$ intervals consisting of consecutive $\mathrm{R}$ waves with sinus rhythm (normal-to-normal R-R interval: $\mathrm{N}-\mathrm{N}$ interval) were obtained. The N-N interval time series thus obtained were analyzed separately for the period of driving in the morning and that during sleeping. For each period, we calculated the following $\mathrm{HRV}$ measures: mean $\mathrm{N}-\mathrm{N}$ interval, standard deviation of all N-N intervals (SDNN), the variances corresponding to ultra-low frequency (ULF; 0-0.0033 Hz), very-low frequency (VLF; 0.0033-0.04 Hz), low frequency (LF; 0.04-0.15 Hz) and high frequency (HF; 0.15-0.40 Hz) bands and $\mathrm{LF} / \mathrm{HF}$. The variances of these frequency components were transformed in the natural logarithmic values ( $\mathrm{Ln})$. We examined the fractal correlation properties of heart rate dynamics using detrended fluctuation analysis (DFA) and calculated the short-term (4 to 11 beat) and long-term ( $\geq 11$ beats) scaling exponents as $\alpha_{1}$ and $\alpha_{2}$, respectively [10]. We also computed the deceleration capacity (DC) by the phase rectified signal averaging of the 24-h N-N interval time series [11].

For the PVT data, RTs were averaged and minor lapse 
frequency was calculated as the percentage of the number of minor lapses in the total number of stimuli over each PVT session.

\section{F. Statistical Analysis}

Statistical analyses system version 9.4 (SAS institute Inc., Cary, NC, USA) was used for the statistical analysis. We used the mixed-model analyses of variance (ANOVA) for repeated measures with color of glasses, age, and sex as the fixed effects and subject as random effect. $P<0.05$ was considered to be statistically significant and Bonferroni adjustment was used to keep type 1 error level in multiple comparisons.

\section{RESULTS}

Among 12 subjects, the duration of morning drive for commuting was $39 \pm 22$ min (median [IQR], 30 [25]-[40] min). Fig. 5 shows the least square means of HRV measures for each color of glasses adjusted for the effects of age and sex.
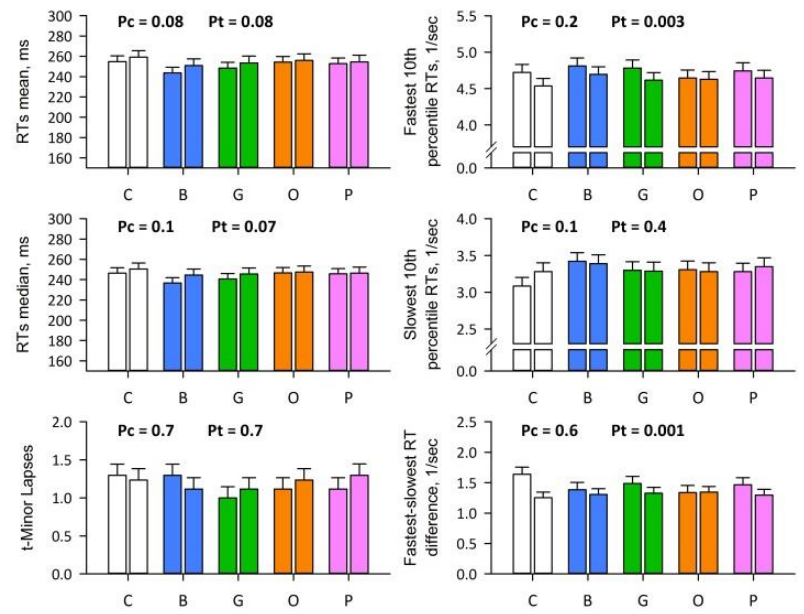

Fig. 6. Effects of color glasses on PVT performance. Right and left bars represent performance of PVT performed at the beginning and the end of working period. $\mathrm{Pc}$ and $\mathrm{Pt}$ indicate significances of the effects of glass color and time, respectively.

Compared with clear glasses, blue glasses during driving increased DC and LF power and decreased HF frequency that reflects respiration frequency. No significant effect was observed for the other colors of glasses.

Fig. 6 shows the effects of color glasses during morning drive on the performance of PVT performed at the beginning and the end of working period. The color of glasses had no significant effect on any measures of PVT performance, while the fastest 10th percentile of RTs was greater (faster) at the benign of work than the end of work. Consequently, the difference between fastest and slowest 10th percentile RTs was greater at the beginning of work than the end of work.

Fig. 7 demonstrates the effects of color glasses on the HRV measures during sleep of that night. No significant effect of color glasses was observed on any HRV measures.

\section{RESULTS}

Among 12 subjects, the duration of morning drive for commuting was $39 \pm 22$ min (median [IQR], 30 [25]-[40] min). Fig. 5 shows the least square means of HRV measures for each color of glasses adjusted for the effects of age and sex.

Compared with clear glasses, blue glasses during driving increased DC and LF power and decreased HF frequency that reflects respiration frequency. No significant effect was observed in other color glasses.

Fig. 6 shows the effects of color glasses during morning drive on the performance of PVT performed at the beginning and the end of working period. The color of glasses had no significant effect on any measures of PVT performance, while the fastest 10th percentile of RTs was greater (faster) at the benign of work than the end of work. Consequently, the difference between fastest and slowest 10th percentile RTs was greater at the beginning of work than the end of work.

Fig. 7 demonstrates the effects of color glasses on the HRV measures during sleep of that night. No significant effect of color glasses was observed on any HRV measures.
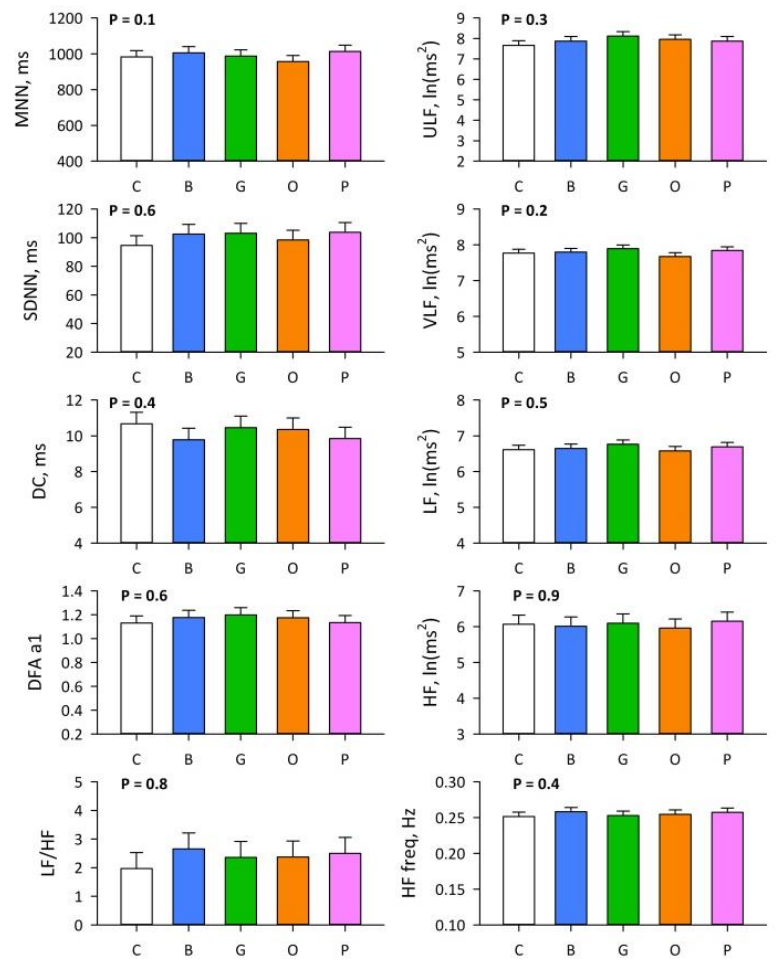

Fig. 7. Effects of color glasses on HRV measures during sleep P values indicate significance of the effect of glass color.

\section{DISCUSSIONS}

This study examined the effects of color glasses during morning drive for commuting on autonomic functions during driving and the after-effects on daytime behavioral alertness during working hours and on nighttime autonomic functions during sleeping at that night in healthy subjects. Compared with clear glasses, blue glasses increased DC and LF power and decreased respiratory frequency during driving. No such effect was observed for green, orange, or pink color glasses. Color glasses during morning drive, however, showed no significant after-effects on the performance of psychomotor vigilance test performed during work hours or on nighttime autonomic function during sleep of that night. These observations suggest that wearing color glasses during driving early in the morning modulates cardiac autonomic functions and respiratory activity during driving, but it has no 
after-effect on daytime behavioral alertness or on autonomic functions during the night of the day.

Given that blue glasses increase the relative but not absolute content of blue wave length light in the light entering the eye, these observations suggest the importance of relative content of blue light for these biological effects. In a previous study using organic light-emitting diode, Yuda et al. [6] demonstrated that exposures to blue light enhances autonomic alertness, while no such effect is observed for white light that contains the same amount of blue light as well as green and red lights. These indicate importance of relative amount of blue-wavelength light for its autonomic effects and support for the utility of blue glasses for that purpose.

The mechanisms for the changes in autonomic indices observed during driving with blue glasses are unclear, because the relationships between HRV measures and autonomic functions are not straight forward. Both DC and LF component of HRV are thought to be modulated by both sympathetic and parasympathetic functions [11], [12]. Because blue glasses showed no significant effects on $\mathrm{HF}$ power that reflects parasympathetic function, the increase in DC and LF power may be attributable to a certain effects on sympathetic function. Given that respiratory frequency reduced during driving with blue glasses, it seemed calm the mind and body during driving with attenuating sympathetic functions.

On the other hand, there are also many possible mechanisms for the reduction of respiratory frequency by blue glasses. Respiration frequency may be controlled by the inputs from both chemoreceptors and baroreceptors and by the psychological factors. Furthermore, the biological effects of blue light could be mediated by non-image forming visual functions which involves intrinsically photosensitive retinal ganglion cells [1], [3], [4], [13], [14]. Blue light is also known to enhance alertness at least immediately after exposure [6]. Further studies are required for determining the biological significance of this finding.

This study has several limitations. The contents of light entering the eyes through color glasses depend on the illuminance and photo-spectral characteristics of the environmental light. Therefore, the results may have been affected by the difference in weather at the time of each experiment and photo-transmissivity characteristics of the window glasses of cars used by individual subjects. The length of driving for commuting differed between subjects, ranging from 20 to $105 \mathrm{~min}$. Thus, the duration for wearing glasses differed between subjects, which may have affected on the results, although the effects did not differ between colors of glasses. Finally, although the first PVT was performed when subjects arrived at their work place, the time from getting off the car to starting PVT was not strictly controlled. If the effects of color glasses on PVT decayed with time, the length of this time may have affected on the results.

\section{CONCLUSIONS}

In conclusion, an increase in the content of blue-wavelength light by wearing blue glasses may attenuate sympathetic function during morning drive for commuting, but it has no after-effect on behavioral alertness during the day or on autonomic functions during the night of the day.

\section{REFERENCES}

[1] C. Cajochen, M. Munch, S. Kobialka, K. Krauchi, R. Steiner, P. Oelhafen et al., "High sensitivity of human melatonin, alertness, thermoregulation, and heart rate to short wavelength light," J Clin Endocrinol Metab, vol. 90, pp. 1311-6, Mar 2005.

[2] S. W. Lockley, E. E. Evans, F. A. Scheer, G. C. Brainard, C. A. Czeisler, and D. Aeschbach, "Short-wavelength sensitivity for the direct effects of light on alertness, vigilance, and the waking electroencephalogram in humans," Sleep, vol. 29, pp. 161-8, Feb 2006.

[3] S. L. Chellappa, R. Steiner, P. Blattner, P. Oelhafen, T. Gotz, and C. Cajochen, "Non-visual effects of light on melatonin, alertness and cognitive performance: can blue-enriched light keep us alert?" PLoS One, vol. 6, p. e16429, 2011.

[4] A. Yasukouchi and K. Ishibashi, "Non-visual effects of the color temperature of fluorescent lamps on physiological aspects in humans," J Physiol Anthropol Appl Human Sci, vol. 24, pp. 41-3, Jan 2005.

[5] E. Yuda, H. Ogasawara, Y. Yoshida, and J. Hayano, "Suppression of vagal cardiac modulation by blue light in healthy subjects," J Physiol Anthropol, vol. 35, p. 24, 2016.

[6] E. Yuda, H. Ogasawara, Y. Yoshida, and J. Hayano, "Enhancement of autonomic and psychomotor arousal by exposures to blue wavelength light: importance of both absolute and relative contents of melanopic component," J Physiol Anthropol, vol. 36, p. 13, Jan 31, 2017.

[7] J. F. Duffy and C. A. Czeisler, "Effect of light on human circadian physiology," Sleep Med Clin, vol. 4, pp. 165-177, Jun 2009.

[8] M. Y. Khitrov, S. Laxminarayan, D. Thorsley, S. Ramakrishnan, S. Rajaraman, N. J. Wesensten et al., "PC-PVT: A platform for psychomotor vigilance task testing, analysis, and prediction," Behav Res Methods, vol. 46, pp. 140-7, Mar 2014.

[9] P. Graw, K. Krauchi, V. Knoblauch, A. Wirz-Justice, and C. Cajochen, "Circadian and wake-dependent modulation of fastest and slowest reaction times during the psychomotor vigilance task," Physiol Behav, vol. 80, pp. 695-701, Feb 2004

[10] C. K. Peng, S. Havlin, H. E. Stanley, and A. L. Goldberger, "Quantification of scaling exponents and crossover phenomena in nonstationary heartbeat time series," CHAOS, vol. 5, pp. 82-7, 1995

[11] A. Bauer, J. W. Kantelhardt, P. Barthel, R. Schneider, T. Makikallio, K. Ulm et al., "Deceleration capacity of heart rate as a predictor of mortality after myocardial infarction: cohort study," Lancet, vol. 367, pp. 1674-1681, 2006.

[12] B. Pomeranz, R. J. Macaulay, M. A. Caudill, I. Kutz, D. Adam, D. Gordon et al., "Assessment of autonomic function in humans by heart rate spectral analysis," Am J Physiol, vol. 248, pp. H151-3, Jan 1985.

[13] D. Litscher, L. Wang, I. Gaischek, and G. Litscher, "The influence of new colored light stimulation methods on heart rate variability, temperature, and well-being: results of a pilot study in humans," Evid Based Complement Alternat Med, vol. 2013, p. 674183, 2013.

[14] V. Daneault, M. Dumont, E. Masse, G. Vandewalle, and J. Carrier, "Light-sensitive brain pathways and aging," J Physiol Anthropol, vol. 35 , p. 9,2016

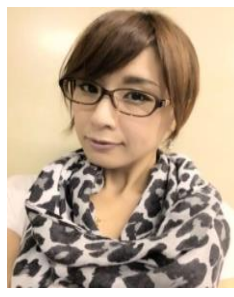

Emi Yuda was born in Mitaka city, Tokyo Japan in 1980. She studied computer science at Moscow University until 2003 and received the M.S. degree from Tsukuba University, Japan. She will receive the $\mathrm{Ph}$.D. degree from Nihon University graduate school From 2013 to 2014 she was a research assistant at the Santa Monica College Computer Science Department in California, USA. Since 2015, she has been a project researcher of New Energy and Industrial Technology Development Organization (NEDO), with the Nagoya City University Graduate School of Medical sciences. Her research is informatics and intellectual property. Since 2017 she has been a member of the Steering Committee of the Electronic Intellectual Property Study Group of Information Processing Society of Japan.

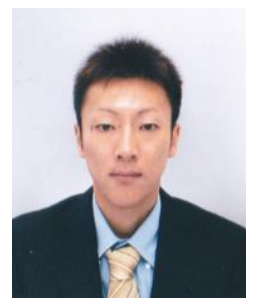

Yutaka Yoshida was born in Aichi, Japan in 1977. He received the B.S. and M.S. degrees in engineering from the Daido University, and Ph.D. degree in information management from Aichi Institute of Technology University, Nagoya, Japan, in 2008. From 2008 to 2012, he was a research assistant with the Nagoya City University Graduate School of Arts and Sciences. From 2012 to 2016, he was a project researcher of "Center of Knowledge", with the Nagoya City University Graduate School of Medical sciences. Since 2016, he has been a research engineer with the Nagoya City University Graduate 
School of Medical sciences. His research interests include bioinformatics engineering and digital signal processing.

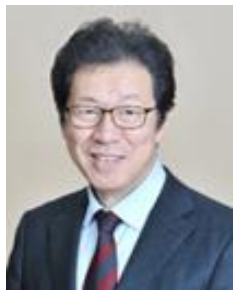

Junichiro Hayano graduated Nagoya City University Medical School, Nagoya, Japan and received M.D. degree in 1980. From 1981 to 1983, he received residency trainings of psychosomatic medicine in Kyushu University School of Medicine, Fukuoka, Japan. He obtained the Ph.D. degree (Dr. of Medical Science) in 1988 from Nagoya City University Graduate School of Medical Sciences. From 1990 to 1991, he was working as a visiting associate at the Behavioral Medicine Research Center, Duke University Medical Center, Durham, NC, USA. In 1984, he got a faculty position at Nagoya City University Medical School and has been a Professor of Medicine at Nagoya City University Graduate School of Medical Sciences since 2003. His current interests are applications of dynamic electrocardiography and bio-signal monitoring to cardiology and health sciences. 